\title{
Prostate Carcinoma Metastatic to the Stomach: Report of Two Cases and Review of the Literature
}

\author{
Adedayo A. Onitilo, MD, MSCR, FACP; Jessica M. Engel, MSN, FNP-BC and Jeffrey M. Resnick, MD
}

\begin{abstract}
We report two cases of prostate carcinoma metastatic to the stomach. In the first case, the patient had indolent disease before presenting with gastric metastasis. The patient responded to treatment with resolution of gastrointestinal bleeding and other symptoms. In the second case, the patient developed aggressive prostate carcinoma while on immunosuppressive therapy following kidney transplant. His tumor became widely systemic, and he ultimately died of his disease. Although rare, the possibility of prostate carcinoma metastatic to the stomach should be considered when a patient with a history of prostatic adenocarcinoma presents with gastrointestinal symptoms.
\end{abstract}

Keywords: Prostate adenocarcinoma, Stomach metastasis, Gastrointestinal adenocarcinoma

Corresponding Author:

Adedayo A. Onitilo, MD, MSCR, FACP

Department of Hematology/Oncology

Marshfield Clinic-Weston Center

3501 Cranberry Boulevard

Weston, WI 54476

Tel.: $715-393-1400$

Fax: 7/5-393-1399

E-mail: onitilo.adedayo@marshfieldclinic.org

Received: March II, 2009

Revised: May 20, 2009

Accepted: May 27, 2009

doi: $10.3|2| / c m r .2010 .855$
$\mathrm{P}$ ostate carcinoma is one of the most frequently diagnosed malignancies in men. ${ }^{1}$ Common sites of metastasis include the lymph nodes and bone; rarely has metastatic prostate carcinoma been reported to involve the stomach ${ }^{2-8}$ or other sites of the upper gastrointestinal tract. ${ }^{9-12}$ Here we describe two unusual cases of prostate carcinoma metastatic to the stomach.

\section{Case I}

An 89-year-old Caucasian man presented with declining health, weakness, nausea, vomiting, and decreased appetite. Comorbidities included hypertension, history of colon polyps, total colectomy following gastrointestinal bleed postcolonoscopy, congestive heart failure, aortic valve replacement, coronary artery disease, and congestive obstructive pulmonary disease. The patient had a 15-year history of elevated and rising prostatic specific antigen (PSA). His PSA a year before presentation was 1565 units. Family history was significant for a brother with prostate carcinoma. The conservative course of annual follow-up had been decided due to the patient's advanced age, two negative prostate biopsies, and lack of symptoms. At the time of presentation the patient's physical health had deteriorated to the extent that he was unable to live independently and had moved into an assisted living facility.

Central to the evaluation of his illness, an esophagogastroduodenal endoscopy showed a poorly distensible stomach with prominent rugal folds and ulcerations distal to the gastroesophageal junction and along the lesser curvature. Due to the markedly elevated serum PSA of 1660 units, the case was discussed with the pathologist. The gastric biopsy (figure 1A) revealed the lamina propria to harbor 

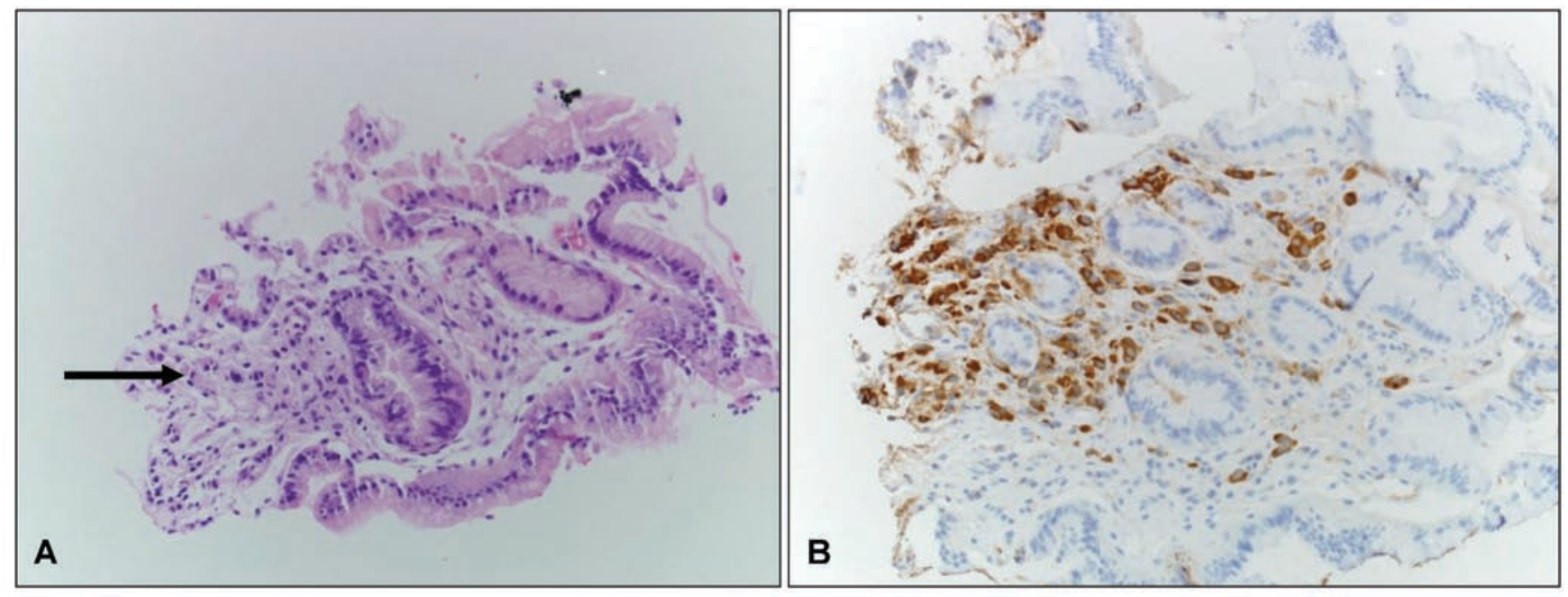

Figure 1: (A) Hematoxylin-eosin stain reveals clusters of monotonous neoplastic cells with round nuclei and amphophilic cytoplasm (see arrow) with no gastric epithelial dysplasia. (B) Immunostain for prostatic-specific antigen demonstrates immunoreactivity with lack of staining by the background mucosal epithelium.

clusters of monotonous neoplastic cells with round nuclei and amphophilic cytoplasm; there was no gastric epithelial dysplasia. The tumor was immunoreactive for PSA (figure 1B) and cytokeratin and negative for chromogranin. A mucicarmine stain was negative. The pathologic findings supported a diagnosis of metastatic prostatic adenocarcinoma.

The staging computed tomography (CT) of the chest/abdomen/ pelvis did not visualize the gastric metastasis well; however, thoracic and lumbar spine and possible omental involvement was detected. The bone scan was also positive for metastatic disease at thoracic (T5) and lumbar (L3) vertebrae. Treatment began immediately with leuprolide $30 \mathrm{mg}$ intramuscular (IM) and bicalutamide $50 \mathrm{mg}$ daily.

At the 1-month follow-up visit, bicalutamide was discontinued, all gastrointestinal symptoms had resolved, and the patient was home functioning independently with activities of daily living, his PSA having dropped from 1660 units to 58.6 units in 1 month (figure 2).

\section{Case 2}

A 57-year-old Caucasian man presented during a routine physical exam with an abnormal digital rectal examination and an elevated PSA (31.54 units). He was on long-term treatment with immunosuppressive agents (cyclosporine, mycophenolate and prednisone) for two prior right kidney transplants (performed for hereditary nephritis 10 years apart). Other comorbidities included hypertension and hypercholesterolemia. His father was diagnosed with prostate carcinoma at age 67.

The prostatic needle core biopsies revealed prostatic adenocarcinoma with Gleason score $9(5+4)$. Bone scan and magnetic resonance imaging (MRI) of the pelvis were negative for metastatic disease. He was treated initially with leuprolide IM. Two months later, intensity modulated radiation therapy (IMRT) was delivered to the prostate and pelvic lymph nodes (total dose $8100 \mathrm{rads} /$ prostate and $4500 \mathrm{rads} /$ lymph nodes).

Approximately 10 months post-diagnosis, the patient developed metastases to the axial and appendicular skeleton, detected by bone scan and confirmed by MRI. He was treated with intravenous zolendronic acid and palliative radiation therapy to both shoulders. He was enrolled in a prospective randomized phase III clinical trial comparing consolidation therapy with or without Strontium-89 following induction chemotherapy in patients with androgen-independent prostate carcinoma. He was treated with estramustine and Taxotere for 3 months, and subsequently with bicalutamide. His disease continued to progress; 15 months from initial diagnosis of prostate carcinoma, both CT and MRI of the head showed left temporal lobe brain metastasis resulting in partial left third nerve palsy, partial trigeminal neuropathy, and left lower extremity weakness and anesthesia.

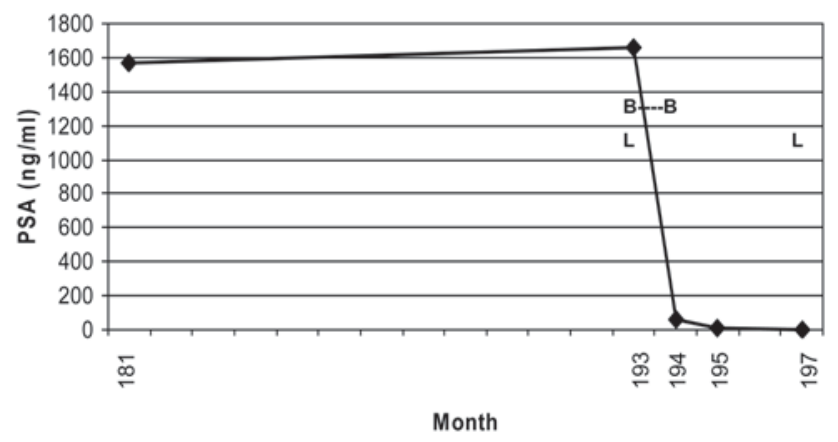

\begin{tabular}{|c|c|c|c|c|c|c|c|c|c|}
\hline & tor & PSA & & & & & & & \\
\hline Month & 0 & 19 & 25 & 128 & 181 & 193 & 194 & 195 & 197 \\
\hline PSA & 9.6 & 15.2 & 17.0 & 273.0 & 1565 & 1660.0 & 58.6 & 10.1 & 3.4 \\
\hline
\end{tabular}

Figure 2: Case 1 PSA history. L, leuprolide; B, bicalutamide. 

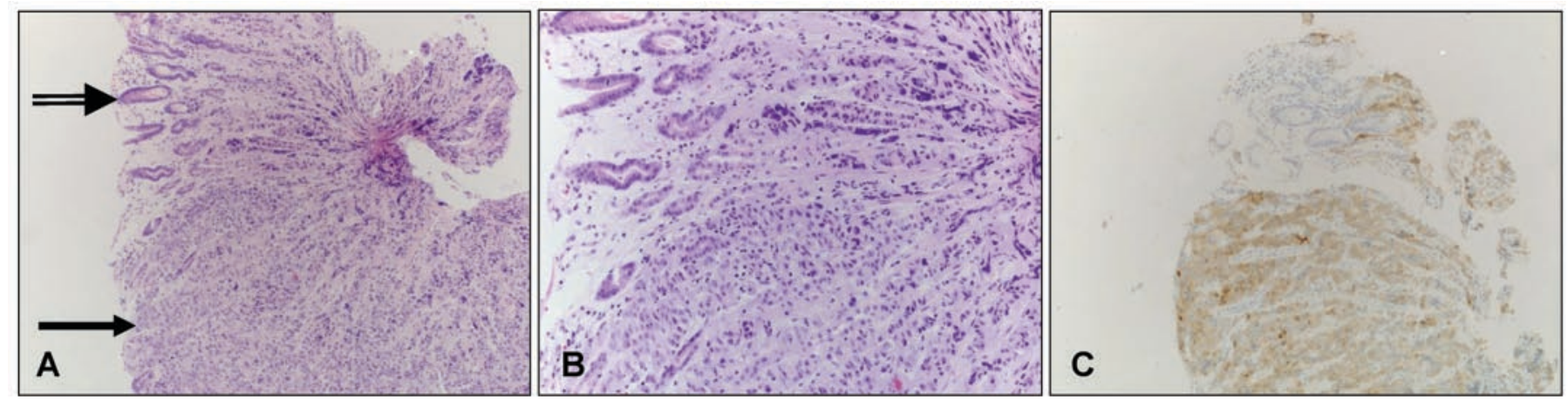

Figure 3: Hematoxylin-eosin stain at low $(A)$ and intermediate $(B)$ magnifications shows neoplastic cells occupying the gastric mucosa in diffuse and nested arrangements (3A, single arrow) and absence of gastric foveolar epithelial dysplasia (3A, double arrow). (C) Lesional cells immunoreactive for PSA.

He subsequently developed hematemesis. Esophagogastroduodenal endoscopy showed a $>5 \mathrm{~cm}$ broad-based ulcerated exophytic lesion in the gastric body. The biopsy (figure 3A and $3 \mathrm{~B}$ ) showed the neoplastic cells to occupy the gastric mucosa in diffuse and nested arrangements; the gastric foveolar epithelium was negative for dysplasia. The lesional cells were immunoreactive for PSA (figure 3C) and cytokeratin and negative for chromogranin. A mucicarmine stain was negative. The features of the adenocarcinoma were in keeping with metastasis from the prostatic primary. The brain metastases were treated with gamma knife. The patient experienced progressive deterioration. About 1-month later, CT of the head showed progressive tumor and hematoma. The patient underwent a left frontoparietal craniotomy evacuation of chronic subdural hematoma and removal of subdural membrane harboring metastatic prostatic adenocarcinoma.

The patient then developed left-sided abdominal pain, and CT of the abdomen and pelvis showed increased adenopathy, two new liver lesions, continued bone metastases, and colonic perforation at the level of splenic flexure. The patient underwent a left hemicolectomy for perforation of a diverticulum. Due to progressive disease and deteriorating condition, he was admitted to hospice care and died 19 months after initial diagnosis of prostate carcinoma. Treatment and PSA levels are shown in figure 4.

\section{Discussion}

We present two unusual cases of prostatic adenocarcinoma metastatic to the stomach, as diagnosed by endoscopic biopsy. In case 1, the patient had indolent disease before presenting with gastric metastasis. The patient responded rapidly and dramatically to treatment with resolution of gastrointestinal bleeding and other symptoms. In case 2, the patient developed very aggressive prostate carcinoma while on immunosuppressive therapy following kidney transplant. His tumor became widely systemic, involving bones, lymph nodes, the central nervous system and the stomach.

Common sites of prostate carcinoma metastasis, in descending order of frequency, include regional lymph nodes, bone, distant lymph nodes, lung, and liver. Prostate carcinoma with gastric metastasis is rare; literature review has revealed only three other cases diagnosed by histology premortem. ${ }^{2-4}$ Four postmortem studies of 67 to 176 patients with metastatic prostate carcinoma reported an incidence of gastric metastasis in $1 \%$ to $4 \%$ of cases. ${ }^{5-8}$ Four case reports describe metastatic disease to nongastric upper gastrointestinal tract sites (3 of the esophagus ${ }^{9-11}$ and 1 of the duodenum ${ }^{12}$ ).

There are two impediments to the recognition of metastatic prostatic adenocarcinoma in gastric mucosal biopsies: (1) the rarity of metastatic prostatic adenocarcinoma involving gastric mucosa concomitant with the unavailability of information to the pathologist regarding the clinical suspicion of metastatic prostatic carcinoma, and (2) the histologic resemblance of metastatic prostatic adenocarcinoma to other epithelial neoplasms more commonly involving the stomach, such as diffuse-type gastric adenocarcinoma or well-differentiated neuroendocrine ("carcinoid") tumor. Besides communication of clinical suspicion to the pathologist, certain histologic features may be helpful in diagnosing metastatic prostatic adenocarcinoma in a gastric biopsy. The absence of gastric epithelial dysplasia raises the consideration of metastasis from an extragastric malignancy, but does not exclude the

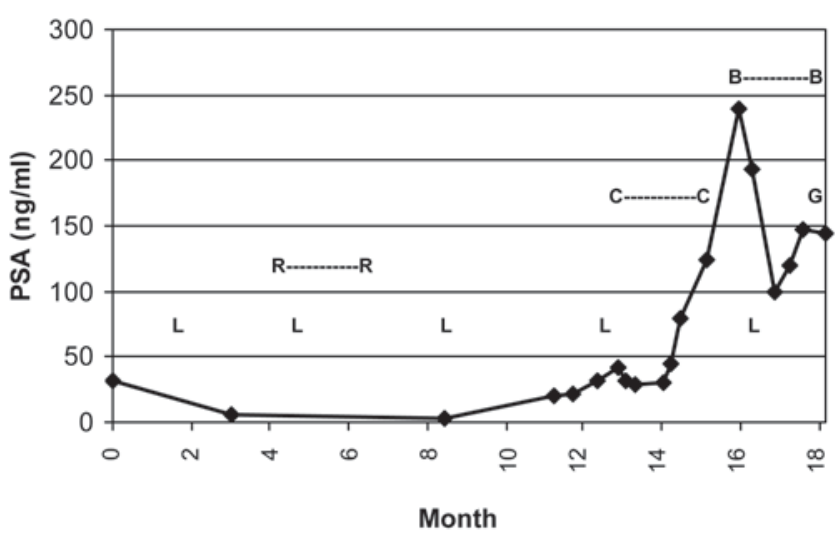

Figure 4: Treatment and PSA levels. L, leuprolide; R, radiation; G, gamma knife; C, chemotherapy; B, bicalutamide. 
possibility of a gastric primary. Relative to the diffuse-type adenocarcinoma for which metastatic adenocarcinoma is most likely to be mistaken, the conventional form of prostatic adenocarcinoma demonstrates relative lack of nuclear anaplasia, with nuclear uniformity, paucity of mitotic figures, and absence of signet-ring cell forms. Furthermore, distinctive from gastric adenocarcinoma, prostatic adenocarcinomas are usually negative for mucicarmine and immunoreactive for PSA. Well-differentiated neuroendocrine tumor may also enter the differential diagnosis; indeed, the oncocytic variant of neuroendocrine tumor (with conspicuous eosinophilic cytoplasm) may feature prominent nucleoli and therefore resemble metastatic prostatic adenocarcinoma. Nevertheless, once the possibility of prostatic adenocarcinoma is entertained, the immunostains for PSA and a neuroendocrine marker (eg, chromogranin) are effective in resolving the differential diagnosis. Epithelioid gastrointestinal stromal tumor may mimic carcinoma histologically, but unlike the latter, is invariably negative for cytokeratin and usually positive for cd117.

Although rare, it is important to consider the possibility of prostate carcinoma metastatic to the gastrointestinal tract in a patient presenting with gastrointestinal symptoms and history of prostatic adenocarcinoma.

\section{Acknowledgements}

The authors thank Marshfield Clinic Research Foundation for its support through the assistance of Marie Fleisner in the preparation of this manuscript.

\section{References}

1. American Cancer Society. Leading sites of new cancer cases and deaths-2008 estimates, Available at:http://www.cancer.org/ downloads/stt/CFF2008M\&F_Sites.pdf. Accessed October 12, 2008.

2. Christoph F, Grünbaum M, Wolkers F, Müller M, Miller K. Prostate cancer metastatic to the stomach. Urology 2004;63:778-779.

3. Ben-Izhak O, Lichtig C. Signet-ring cell carcinoma of the prostate mimicking primary gastric carcinoma. J Clin Pathol 1992;45:452-454.

4. Holderman WH, Jacques JM, Blackstone MO, Brasitus TA. Prostate cancer metastatic to the stomach: clinical aspects and endoscopic diagnosis. J Clin Gastroenterol 1992; 14:251-254

5. Green LK. Hematogenous metastases to the stomach. A review of 67 cases. Cancer 1990;65:1596-1600.

6. Oda I, Kondo H, Yamao T, Saito D, Ono H, Gotoda T, Yamaguchi $\mathrm{H}$, Yoshida S, Shimoda T. Metastatic tumors to the stomach: analysis of 54 patients diagnosed at endoscopy and 347 autopsy cases. Endoscopy 2001;33:507-510.

7. Arnheim F. Carcinoma of the prostate: a study of post-mortem findings in 176 cases. J Urol 1948;60:599-603.

8. Mintz ER, Smith GG. Autopsy findings in 100 cases of prostate carcinoma. N Eng J Med 1934;211:479-487.

9. Nakamura T, Mohri H, Shimazaki M, Ito Y, Ohnishi T, Nishino Y. Esophageal metastasis from prostate cancer: diagnostic use of reverse transcriptase-polymerase chain reaction for prostate-specific antigen. J Gastroenterol 1997;32:236-240.
10. Gore RM, Sparberg M. Metastatic carcinoma of the prostate to the esophagus. Am J Gastroenterol 1982;77:358-359.

11. Eaves R, Lambert J, Rees J, King RW. Achalasia secondary to carcinoma of the prostate. Dig Dis Sci 1983;28:278-284.

12. Malhi-Chowla N, Wolfsen HC, Menke D, Woodward TA. Prostate cancer metastasizing to the small bowel. J Clin Gastroenterol 2001;32:439-440.

\section{Author Affiliations}

Adedayo A. Onitilo, MD, MSCR, FACP*; Jessica M. Engel, MSN, FNP-BC+'; Jeffrey M. Resnick, $M D^{\dagger}$

*Department of Hematology/Oncology, Marshfield ClinicWeston Center, Weston, Wisconsin

tDepartment of Hematology/Oncology, Marshfield Clinic, Marshfield, Wisconsin

$¥$ Department of Pathology, Marshfield Labs, Marshfield

Clinic, Marshfield, Wisconsin 